\title{
A short synthesis of (+)- $\beta$-lycorane by asymmetric conjugate addition cascade
}

\section{$\operatorname{AUTHOR}(\mathrm{S})$ :}

Nishimura, Katsumi; Fukuyama, Naoshi; Yasuhara, Tomohisa; Yamashita, Mitsuaki; Sumiyoshi, Takaaki;

Yamamoto, Yasutomo; Yamada, Ken-ichi; Tomioka, Kiyoshi

\section{CITATION:}

Nishimura, Katsumi ...[et al]. A short synthesis of (+)- $\beta$-lycorane by asymmetric conjugate addition cascade. Tetrahedron 2015, 71(39): 7222-7226

\section{ISSUE DATE:}

2015-09-30

URL:

http://hdl.handle.net/2433/201854

\section{RIGHT:}

(c) 2015. This manuscript version is made available under the CC-BY-NC-ND 4.0 license

http://creativecommons.org/licenses/by-nc-nd/4.0/; The full-text file will be made open to the public on 30 September 2017 in accordance with publisher's 'Terms and Conditions for Self-Archiving'.; この論文は出版社版でありません。引 用の際には出版社版をご確認ご利用ください。; This is not the published version. Please cite only the published version. 


\title{
A short synthesis of (+)- $\beta$-lycorane by asymmetric conjugate addition cascade
}

\author{
Katsumi Nishimura ${ }^{a}$, Naoshi Fukuyama ${ }^{a}$, Tomohisa Yasuhara ${ }^{a}$, Mitsuaki Yamashita ${ }^{a}$, Takaaki Sumiyoshi ${ }^{a}$, \\ Yasutomo Yamamoto ${ }^{\mathrm{b}}$, Ken-ichi Yamada ${ }^{\mathrm{a}}$, and Kiyoshi Tomioka ${ }^{\mathrm{a}, \mathrm{b}},{ }_{*}$
}

${ }^{a}$ Graduate School of Pharmaceutical Sciences, Kyoto University, Yoshida, Sakyo-ku, Kyoto 606-8501, Japan

${ }^{b}$ Faculty of Pharmaceutical Sciences, Doshisha Women's College of Liberal Arts, Kodo, Kyotanabe 610-0395, Japan

\section{ARTICLE INFO}

Article history:

Received

Received in revised form

Accepted

Available online

\section{Keywords:}

chiral ligand

asymmetric synthesis

organolithiums

conjugate addition

lycorane

\section{ABSTRACT}

The chiral diether ligand-controlled asymmetric conjugate addition of organolithiums to nona2,7-dienedioate and subsequent intramolecular conjugate addition of the enolate intermediate gave all-trans trisubstituted cyclohexanes with high ee and yields. Using this methodology, an efficient short asymmetric total synthesis of $(+)-\beta$-lycorane was accomplished in $33 \%$ overall yield through five steps from the dienedioate.

2009 Elsevier Ltd. All rights reserved.

This is an author version based on a template provided by Elsevier.

\section{Introduction}

The Amaryllidaceae alkaloids have attracted much attention due to their rich bioactivities, such as anti-Alzheimer, antifungal, antibacterial, and antineoplastic activities. ${ }^{1}$ Among these alkaloids, lycorine $(\mathbf{1})^{1,2}$ and its deoxygenated derivatives lycoranes $(\mathbf{2 a} \text { and } \mathbf{b})^{3}$ possess the characteristic pyrrolophenanthridine skeleton (Figure 1). We have previously reported the asymmetric total synthesis of (-)-lycorine (1) using a chiral ligand-controlled asymmetric conjugate addition cascade. ${ }^{4,5}$ As a continuation of this study to show the utility of this cascade reaction, here we report a short asymmetric total synthesis of $(+)-\beta$-lycorane (2b). ${ }^{6,7,8}$

Our strategy starts from the asymmetric conjugate addition ${ }^{9,10}$ of aryllithium 4 to nona-2,7-dienedioate $3^{11,12}$ mediated by chiral ligand 5 (Scheme 1). The subsequent intramolecular Michael reaction of the resulting enolate $\mathbf{6}$ would afford trisubstituted cyclohexane $7 \mathbf{a}$ with three contiguous stereogenic centers. ${ }^{4}$ The nitrogen functionality could be introduced by Curtius rearrangement, and the subsequent cyclization gives $(+)-\beta$-lycorane $(\mathbf{2 b})$.



(-)-lycorine (1)

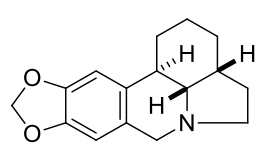

$(+)$-[?-lycorane (2a)

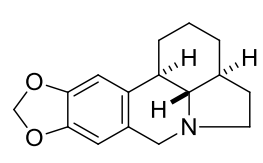

$(+)$-- -lycorane (2b)

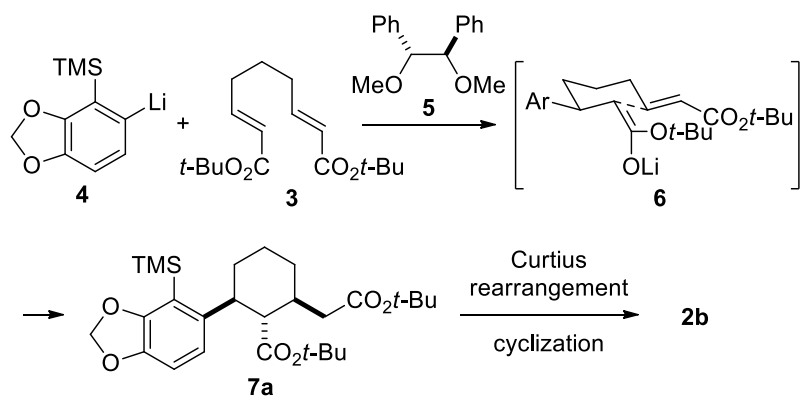

Scheme 1. Synthetic strategy to (+)- $\beta$-lycorane.

\section{Results and discussion}

First, the reaction of dienedioate $\mathbf{3}$ and phenyllithium was investigated as a model reaction. The reaction of $\mathrm{PhLi}(3$ equiv) in the presence of 5 (4.2 equiv) was complete within $0.5 \mathrm{~h}$ to give trans,trans-cyclohexane $\boldsymbol{t t}$-7b with $71 \%$ ee and trans,ciscyclohexane $\boldsymbol{t} \boldsymbol{c}-\mathbf{7 b}$ with $3 \%$ ee in $50 \%$ and $9 \%$ yields, respectively (Table 1 , entry 1). The relative configuration of $\boldsymbol{t} \boldsymbol{t}$-7 $\mathbf{b}$ was assigned based on the trans-diaxial coupling between the two pairs of the adjacent methine protons $(J=11.2 \mathrm{~Hz}$ each). The absolute configuration of $\boldsymbol{t} \boldsymbol{t}-\mathbf{7} \mathbf{b}$ was determined by conversion to a known compound with established stereochemistry (see Supplementary Material).

Figure 1. Structures of lycorine and lycoranes. 
Table 1. Asymmetric conjugate addition cascade. ${ }^{a}$



${ }^{a}$ All reactions were carried out using $\operatorname{ArLi}\left(3\right.$ equiv) and $\mathbf{5}$ (4.2 equiv). ${ }^{b}$ The ee was determined after derivatization (see Supplementary Material).

Toward the synthesis of (+)- $\beta$-lycorane, benzodioxol-5yllithium was employed in the cascade reaction. The reaction proceeded with comparable enantioselectivity, and $\boldsymbol{t t}$-7f with $74 \%$ ee was obtained in $40 \%$ yield along with $\boldsymbol{t c}$-7f with $72 \%$ ee

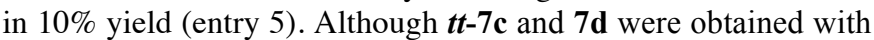
the same level of enantioselectivity $(67-68 \%$ ee) in the reactions with 2-methyl- and 2-isopropylphenyllithiums (entries 2 and 3), the enantioselectivity was improved in the reaction with 1naphthyllithium to give $\boldsymbol{t t}$-7e with $81 \%$ ee in $79 \%$ yield along with $\boldsymbol{t c}$-7e with $39 \%$ ee in $4 \%$ yield (entry 4 ). Encouraged by this result, we utilized aryllithium 4, bearing a bulky TMS group at the ortho-position, in the reaction. To our delight, $\boldsymbol{t t}$-7a, which has all the carbon atoms and stereochemistry required for the total synthesis, was obtained in $68 \%$ yield with almost perfect enantioselectivity (99\% ee; entry 6). Interestingly, the products were obtained in higher yields with the more bulky aryllithiums (entries 1 and 5 vs $2-4$ and 6). This is explainable by decreased order of aggregation of the organolithium compounds in solution by the steric hindrance. ${ }^{13}$ Unfortunately, lowering the amount of the aryllithium, the enantiomeric excesses of $\boldsymbol{t} \boldsymbol{t}-\mathbf{7} \mathbf{a}$ and $\boldsymbol{t} \boldsymbol{c}-\mathbf{7} \mathbf{\mathbf { a }}$ were decreased to $85 \%$ and $54 \%$ ( 2 equiv), or to $50 \%$ and $27 \%(1.2$ equiv), respectively, although the yield and the diastereoselectivity were unchanged. These results indicate that the lithium enolate that was generated by the conjugate addition cascade would form a ternary complex with the aryllithium and chiral ligand, which undergoes less enantioselective conjugate addition. ${ }^{14}$ Importantly, chiral ligand $\mathbf{5}$ was quantitatively recovered without any loss of optical purity, and was reusable.

The diastereoselectivity of the cascade reaction is explainable as shown in Scheme 2. The lithium enolate intermediate, which results from the conjugate addition of $\mathbf{4}$ to $\mathbf{3}$, would undergo the intramolecular Michael reaction preferentially via conformer A and lead to $\mathbf{t t - 7 a}$, due to the absence of the 1,3-diaxial repulsion of the alkenoate moiety, which exists in conformer $\mathbf{B}$. Therefore, the lower diastereoselectivity $(4: 1$, Table 1 , entry 6$)$ than that in the previously reported reaction of $\mathbf{4}$ and $\mathbf{8}$ to give $\mathbf{9}(9: 1)^{4}$ could be attributed to the decreased 1,3-diaxial repulsion in conformer B $(\mathrm{R}=\mathrm{H}$ vs ethylenedioxy).

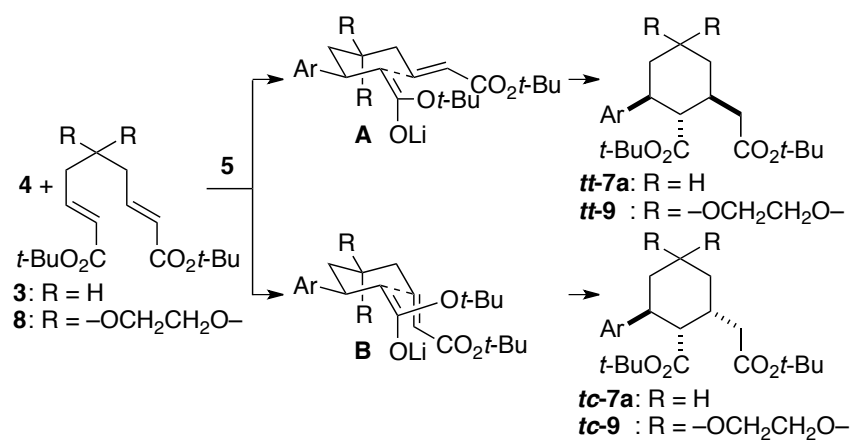

Scheme 2. Rationale for observed diastereoselectivity.

The asymmetric total synthesis of (+)- $\beta$-lycorane (2b) was accomplished starting from $\boldsymbol{t} \boldsymbol{t}-\mathbf{7 a}$ as shown in Scheme 3. Treatment of $\boldsymbol{t t}$-7a with $\mathrm{HCl}$ for $0.5 \mathrm{~h}$ in refluxing ethanol gave protodesilylated half ester $\mathbf{1 0}$ in $98 \%$ yield. Interestingly and usefully, the carboxylic acid at the $\mathrm{C}-2$ position was not esterified, probably due to steric hindrance. ${ }^{15}$ Curtius rearrangement using diphenylphosphoryl azide (DPPA) ${ }^{16}$ followed by Bischler-Napieralskitype cyclization with polyphosphoric acid (PPA) ${ }^{17}$ converted 10 into lactam 11 via an isocyanate intermediate in $70 \%$ yield. Finally, treatment of $\mathbf{1 1}$ with borane-dimethyl sulfide complex ${ }^{18}$ in refluxing THF induced three sequential transformations: reduction of the lactam, lactam formation between the resulting amine and the ester moiety, and reduction of the resulting lactam to directly give (+)- $\beta$-lycorane $(\mathbf{2 b})$ in $70 \%$ yield. ${ }^{1} \mathrm{H}$ and ${ }^{13} \mathrm{C}$ NMR, ${ }^{6 c, d}$ and the specific rotation ${ }^{6 \mathrm{~d}}$ were in good agreement with those reported. Notably, the asymmetric total synthesis was accomplished through only five steps from $\mathbf{3}$ in high overall yield $(33 \%)$.


Scheme 3. Asymmetric total synthesis of (+)- $\beta$-lycorane (2b)

\section{Conclusion}

This study revealed that the bulky TMS group at the orthoposition of aryllithium is effective to improve the enantioselectivity of the chiral ligand-mediated asymmetric conjugate addition cascade of nonadienedioate. Cyclohexanes bearing three contiguous substituents with trans,trans-configuration were obtained in high optical purity. It was suggested that the 1,3-diaxial interaction would play an important role in determining the diastereoselectivity. This methodology enables the formation of two $\mathrm{C}-\mathrm{C}$ bonds and three stereogenic centers in one pot to give synthetically useful chiral cyclohexane derivatives. The utility of this methodology was clearly demonstrated by the achievement of the shortest asymmetric total synthesis of $(+)-\beta$-lycorane. Importantly, the chiral ligand could be recycled, and the one-pot reactions are economically and ecologically beneficial. 


\section{Experimental section}

\subsection{General}

All melting points are uncorrected. Silica gel was used for column chromatography. ${ }^{1} \mathrm{H}$ and ${ }^{13} \mathrm{C}$ NMR (500 and $125 \mathrm{MHz}$, respectively) were measured in $\mathrm{CDCl}_{3}$ unless otherwise mentioned. Chemical shifts and coupling constants are presented in ppm $\delta$ relative to tetramethylsilane and $\mathrm{Hz}$, respectively. The wavenumbers of maximum absorption peaks of IR spectroscopy are presented in $\mathrm{cm}^{-1}$. Chiral ligand $\mathbf{5}$ was prepared as previously described. ${ }^{19}$

4.1.1. Di-tert-butyl (2E,7E)-nona-2,7-dienedioate $(3):{ }^{20}$ To a stirred suspension of tert-butyl triphenylphosphoranylideneacetate $(161 \mathrm{~g}, 0.43 \mathrm{~mol})$ in toluene $(0.26 \mathrm{~L})$ was added a solution of glutaraldehyde $(17.2 \mathrm{~g}, 0.17 \mathrm{~mol})$ in toluene $(80 \mathrm{~mL})$ at $\mathrm{rt}$. After $21 \mathrm{~h}$, the mixture was filtered, and the residue was washed with hexane. The combined filtrate and washings were concentrated and purified by column chromatography (hexane/EtOAc $=20 / 1$ ) to give the title compound $(53 \mathrm{~g}, 90 \%)$ as a colorless oil of bp 135-140 ${ }^{\circ} \mathrm{C} / 0.2 \mathrm{mmHg}: R_{f}=0.4$ (hexane/EtOAc $=9 / 1$ ). ${ }^{1} \mathrm{H}$ NMR: $1.48(18 \mathrm{H}, \mathrm{s}), 1.62(2 \mathrm{H}$, quintet, $J=7.3), 2.20$ (4H, ddt, $J$ $=1.5,7.0,7.3), 5.75(2 \mathrm{H}, \mathrm{dt}, J=15.6,1.5), 6.83(2 \mathrm{H}, \mathrm{dt}, J=15.6$, 7.0). ${ }^{13} \mathrm{C}$ NMR: $26.4\left(\mathrm{CH}_{2}\right), 28.1\left(\mathrm{CH}_{3}\right), 31.2\left(\mathrm{CH}_{2}\right), 80.1(\mathrm{C})$, 123.6 (CH), $146.8(\mathrm{CH}), 165.9(\mathrm{C})$. IR (neat): 1710, 1650. EIMS $m / z: 223\left(\mathrm{M}-t\right.$-BuO). Anal. Calcd for $\mathrm{C}_{17} \mathrm{H}_{28} \mathrm{O}_{4}: \mathrm{C}, 68.89 ; \mathrm{H}$, 9.52. Found: $\mathrm{C}, 68.87 ; \mathrm{H}, 9.57 .{ }^{1} \mathrm{H}$ NMR and IR spectra were in agreement with those reported, ${ }^{20}$ while ${ }^{13} \mathrm{C}$ NMR had slight difference; $26.4\left(\mathrm{CH}_{2}\right)$ instead of reported $29.7\left(\mathrm{CH}_{2}\right)$.

\subsection{Asymmetric Conjugate Addition Cascade (Table 1)}

4.2.1. General Procedure. $(1 S, 2 S, 3 S)$ - and $(1 R, 2 S, 3 S)$-tertButyl 2-tert-butoxycarbonyl-3-(4-trimethylsilylbenzo[1,3]dioxol-5-yl)cyclohexaneacetate (tt-7a and $t c-7 a)$ (Table 1 , entry 3): To a solution of $5(10.2 \mathrm{~g}, 42 \mathrm{mmol})$ and 3,4-methylenedioxy2-trimethylsilylbromobenzene ${ }^{21}(8.19 \mathrm{~g}, 30 \mathrm{mmol})$ in toluene $(280 \mathrm{~mL})$ was added a solution of $t$-BuLi $(1.69 \mathrm{M} ; 16.6 \mathrm{~mL}, 28$ mmol) in pentane at $-78{ }^{\circ} \mathrm{C}$, and the resulting solution was stirred for $1 \mathrm{~h}$ at the same temperature. A solution of $\mathbf{3}(2.96 \mathrm{~g}, 10$ mmol) in toluene $(20 \mathrm{~mL})$ was added at $-78^{\circ} \mathrm{C}$, and the mixture was stirred for $10 \mathrm{~min}$ at the same temperature. The reaction was quenched by the addition of saturated $\mathrm{NH}_{4} \mathrm{Cl}$. The organic layer was separated, and the aqueous layer was extracted with $\mathrm{Et}_{2} \mathrm{O}$. The combined organic layers were successively washed with saturated $\mathrm{NaHCO}_{3}$ and brine, dried over $\mathrm{Na}_{2} \mathrm{SO}_{4}$, and concentrated. Column chromatography (hexane $/ \mathrm{Et}_{2} \mathrm{O}=30 / 1$ ) gave $\boldsymbol{t} \boldsymbol{t}-\mathbf{7} \mathbf{a}$ $(3.31 \mathrm{~g}, 68 \%)$ with $99 \%$ ee and $\boldsymbol{t c}-\mathbf{7 a}(860 \mathrm{mg}, 18 \%)$ with $88 \%$ ee as colorless oils, and $\mathbf{5}$ (10.2 g, quantitative recovery) as a colorless solid.

tt-7a: $R_{f}=0.4$ (hexane/ $/ \mathrm{Et}_{2} \mathrm{O}=9 / 1$, developed twice). $[\alpha]_{\mathrm{D}}{ }^{25}$ +16.8 (c 1.26, $\left.\mathrm{CHCl}_{3}\right)$. ${ }^{1} \mathrm{H}$ NMR: $0.35(9 \mathrm{H}, \mathrm{s}), 1.07(9 \mathrm{H}, \mathrm{s}), 1.07-$ $1.20(1 \mathrm{H}, \mathrm{m}), 1.20-1.34(2 \mathrm{H}, \mathrm{m}), 1.42(9 \mathrm{H}, \mathrm{s}), 1.72-1.82(2 \mathrm{H}, \mathrm{m})$, $1.89(1 \mathrm{H}, \mathrm{m}), 2.01(1 \mathrm{H}, \mathrm{dd}, J=9.8,14.4), 2.05(1 \mathrm{H}, \mathrm{m}), 2.27(1 \mathrm{H}$, $\mathrm{dd}, J=2.4,14.4), 2.33(1 \mathrm{H}, \mathrm{t}, J=11.0), 2.97(1 \mathrm{H}, \mathrm{dt}, J=1.8$, 11.0), $5.78(2 \mathrm{H}, \mathrm{m}), 6.68-6.76(2 \mathrm{H}, \mathrm{m}) .{ }^{13} \mathrm{C} \mathrm{NMR}: 1.5\left(\mathrm{CH}_{3}\right)$, $25.5\left(\mathrm{CH}_{2}\right), 27.6\left(\mathrm{CH}_{3}\right), 28.0\left(\mathrm{CH}_{3}\right), 30.6\left(\mathrm{CH}_{2}\right), 36.1\left(\mathrm{CH}_{2}\right), 37.6$ $(\mathrm{CH}), 40.2\left(\mathrm{CH}_{2}\right), 45.4(\mathrm{CH}), 55.4(\mathrm{CH}), 79.6(\mathrm{C}), 80.1(\mathrm{C}), 99.4$ $\left(\mathrm{CH}_{2}\right), 108.5(\mathrm{CH}), 119.0(\mathrm{C}), 120.1(\mathrm{CH}), 142.5(\mathrm{C}), 144.0(\mathrm{C})$, 152.2 (C), 171.4 (C), 173.3 (C). IR (neat): 1728. EIMS $m / z: 490$ $\left(\mathrm{M}^{+}\right)$. Anal. Calcd for $\mathrm{C}_{27} \mathrm{H}_{42} \mathrm{O}_{6} \mathrm{Si}: \mathrm{C}, 66.09 ; \mathrm{H}, 8.63$. Found: $\mathrm{C}$, $65.92 ; \mathrm{H}, 8.70$. The ee was determined by HPLC (Daicel Chiralcel OD-H, hexane $/ i-\mathrm{PrOH}=1000 / 1,1 \mathrm{~mL} / \mathrm{min}, 250 \mathrm{~nm}$; major $9.5 \mathrm{~min}$, minor $7.5 \mathrm{~min}$ ).

tc-7a: $R_{f}=0.5$ (hexane $/ \mathrm{Et}_{2} \mathrm{O}=9 / 1$, developed twice). $[\alpha]_{\mathrm{D}}{ }^{25}$ $+11.7\left(c\right.$ 1.32, $\left.\mathrm{CHCl}_{3}\right) .{ }^{1} \mathrm{H}$ NMR: $0.41(9 \mathrm{H}, \mathrm{s}), 1.18(9 \mathrm{H}, \mathrm{s}), 1.1-$ 1.3 (2H. m), 1.44 (9H, s), 1.52-1.62 (2H, m), 1.72-1.84 (2H, m),
$2.38(1 \mathrm{H}, \mathrm{dd}, J=4.6,15.2), 2.53(1 \mathrm{H}, \mathrm{dd}, J=3.7,15.2), 2.71$ $(1 \mathrm{H}, \mathrm{m}), 2.84(1 \mathrm{H}, \mathrm{dd}, J=4.0,11.8), 3.00(1 \mathrm{H}, \mathrm{dt}, J=3.1,11.8)$, $5.83(2 \mathrm{H}, \mathrm{m}), 6.66(1 \mathrm{H}, \mathrm{d}, J=8.2), 6.71(1 \mathrm{H}, \mathrm{d}, J=8.2) .{ }^{13} \mathrm{C}$ NMR: $1.6\left(\mathrm{CH}_{3}\right), 20.5\left(\mathrm{CH}_{2}\right), 27.8\left(\mathrm{CH}_{3}\right), 28.1\left(\mathrm{CH}_{3}\right), 29.4\left(\mathrm{CH}_{2}\right)$, $33.3(\mathrm{CH}), 34.2\left(\mathrm{CH}_{2}\right), 37.0\left(\mathrm{CH}_{2}\right), 38.5(\mathrm{CH}), 53.0(\mathrm{CH}), 79.7$ (C), $80.0(\mathrm{C}), 99.6\left(\mathrm{CH}_{2}\right), 108.6(\mathrm{CH}), 118.0(\mathrm{CH}), 118.9(\mathrm{C})$, 143.9 (C), $144.4(\mathrm{C}), 152.5$ (C), $172.4(\mathrm{C}), 172.6$ (C). IR (neat): 1728. EIMS m/z: $490\left(\mathrm{M}^{+}\right)$. Anal. Calcd for $\mathrm{C}_{27} \mathrm{H}_{42} \mathrm{O}_{6} \mathrm{Si}: \mathrm{C}, 66.09$; H, 8.63. Found: C, 66.17; H, 8.70. The ee was determined by HPLC (Daicel Chiralcel OD-H, hexane $/ i-\mathrm{PrOH}=1000 / 1,1$ $\mathrm{mL} / \mathrm{min}, 250 \mathrm{~nm}$; major $8.9 \mathrm{~min}$, minor $7.5 \mathrm{~min}$ ).

4.2.2. tert-Butyl (1S,2S,3S)-2-(tert-butoxycarbonyl)-3-phenylcyclohexaneacetate (tt-7b) (Table 1, entry 1): $R_{f}=0.5$ (hexane/ EtOAc $=9 / 1)$. A white solid of mp $70-72{ }^{\circ} \mathrm{C} .71 \%$ ee. $[\alpha]_{\mathrm{D}}{ }^{25}$ $+14.0\left(\right.$ c 5.00, $\left.\mathrm{CHCl}_{3}\right) .{ }^{1} \mathrm{H}$ NMR $\left(\mathrm{C}_{6} \mathrm{D}_{6}\right): 1.12(9 \mathrm{H}, \mathrm{s}), 1.22-1.38$ $(3 \mathrm{H}, \mathrm{m}), 1.41(9 \mathrm{H}, \mathrm{s}), 1.60(1 \mathrm{H}, \mathrm{m}), 1.70(1 \mathrm{H}, \mathrm{m}), 2.02(1 \mathrm{H}, \mathrm{m})$, $2.24(1 \mathrm{H}, \mathrm{dd}, J=9.5,15.0), 2.34(1 \mathrm{H}, \mathrm{t}, J=11.2), 2.40(1 \mathrm{H}, \mathrm{m})$, $2.60(1 \mathrm{H}, \mathrm{dd}, J=3.1,15.0), 2.81(1 \mathrm{H}, \mathrm{dt}, J=3.4,11.2), 7.05-$ $7.20(5 \mathrm{H}, \mathrm{m}) .{ }^{13} \mathrm{C}$ NMR $\left(\mathrm{C}_{6} \mathrm{D}_{6}\right): 26.0\left(\mathrm{CH}_{2}\right), 27.2\left(\mathrm{CH}_{3}\right), 28.1$ $\left(\mathrm{CH}_{3}\right), 31.2\left(\mathrm{CH}_{2}\right), 34.4\left(\mathrm{CH}_{2}\right), 37.9(\mathrm{CH}), 40.7\left(\mathrm{CH}_{2}\right), 48.3(\mathrm{CH})$, $56.1(\mathrm{CH}), 79.5(\mathrm{C}), 79.8(\mathrm{C}), 126.6(\mathrm{CH}), 128.3(\mathrm{CH}), 128.3$ $(\mathrm{CH}), 144.6(\mathrm{C}), 171.2(\mathrm{C}), 173.1(\mathrm{C})$. IR $\left(\mathrm{CHCl}_{3}\right)$ : 1710. EIMS $\mathrm{m} / z: 374\left(\mathrm{M}^{+}\right), 318,262$. Anal. Calcd for $\mathrm{C}_{23} \mathrm{H}_{34} \mathrm{O}_{4}: \mathrm{C}, 73.76 ; \mathrm{H}$, 9.15. Found: $\mathrm{C}, 73.75$; H, 9.33. The ee was determined after reduction with $\mathrm{LiAlH}_{4}$. The absolute configuration was determined after conversion into a known compound (see Supplementary Material).

4.2.3. tert-Butyl $(1 R, 2 S, 3 S)$-2-(tert-butoxycarbonyl)-3-phenylcyclohexaneacetate $(t c-7 b)$ (Table 1 , entry 1): $R_{f}=0.6$ (hexane/ EtOAc $=9 / 1)$. A white solid of mp $90-95{ }^{\circ} \mathrm{C} .3 \%$ ee. $[\alpha]_{\mathrm{D}}{ }^{25}-19.8$ (c 2.04, $\left.\mathrm{CHCl}_{3}\right) .{ }^{1} \mathrm{H}$ NMR: $1.15(9 \mathrm{H}, \mathrm{s}), 1.34-1.40(1 \mathrm{H}, \mathrm{m}), 1.45$ $(9 \mathrm{H}, \mathrm{s}), 1.58-1.62(3 \mathrm{H}, \mathrm{m}), 1.79(1 \mathrm{H}, \mathrm{m}), 1.87(1 \mathrm{H}, \mathrm{m}), 2.45(1 \mathrm{H}$, $\mathrm{dd}, J=9.8,15.6), 2.50(1 \mathrm{H}, \mathrm{dd}, J=4.9,15.6), 2.73(1 \mathrm{H}, \mathrm{m}), 2.82$ $(2 \mathrm{H}, \mathrm{m}), 7.15-7.25(5 \mathrm{H}, \mathrm{m}) .{ }^{13} \mathrm{C}$ NMR: $20.6\left(\mathrm{CH}_{2}\right), 27.7\left(\mathrm{CH}_{3}\right)$, $28.1\left(\mathrm{CH}_{3}\right), 29.5\left(\mathrm{CH}_{2}\right), 32.8(\mathrm{CH}), 34.2\left(\mathrm{CH}_{2}\right), 35.0\left(\mathrm{CH}_{2}\right), 40.9$ $(\mathrm{CH}), 52.6(\mathrm{CH}), 80.1(\mathrm{C}), 80.2(\mathrm{C}), 126.1(\mathrm{CH}), 127.4(\mathrm{CH})$, 128.1 (CH), $145.2(\mathrm{C}), 172.4(\mathrm{C}), 172.9(\mathrm{C})$. IR $\left(\mathrm{CHCl}_{3}\right): 1710$. EIMS $m / z: 374\left(\mathrm{M}^{+}\right), 318,262$. Anal. Calcd for $\mathrm{C}_{23} \mathrm{H}_{34} \mathrm{O}_{4}: \mathrm{C}$, 73.76; H, 9.15. Found: C, 73.89; H, 9.00. The ee was determined after conversion to the corresponding dimethyl ester (see Supplementary Material).

4.2.4. (1S,2S,3S)-tert-Butyl 2-(tert-butoxycarbonyl)-3-(2-methylphenyl)cyclohexanacetate (tt-7c) (Table 1, entry 2): $R_{f}=0.5$ (hexane $/ \mathrm{Et}_{2} \mathrm{O}=9 / 1$, developed twice). A colorless oil. $68 \%$ ee (HPLC: Daicel Chiralcel OD-H $\times 2$ were connected, hexane $/ i$ $\mathrm{PrOH}=100 / 1,1 \mathrm{~mL} / \mathrm{min}, 254 \mathrm{~nm}$; major $37.5 \mathrm{~min}$, minor 33.2 min). $[\alpha]_{\mathrm{D}}{ }^{25}+13.4\left(c 2.16, \mathrm{CHCl}_{3}\right) .{ }^{1} \mathrm{H}$ NMR: $1.07(9 \mathrm{H}, \mathrm{s}), 1.17$ $(1 \mathrm{H}, \mathrm{m}), 1.33(1 \mathrm{H}, \mathrm{m}), 1.46(9 \mathrm{H}, \mathrm{s}), 1.56(1 \mathrm{H}, \mathrm{m}), 1.81(2 \mathrm{H}, \mathrm{m})$, $1.94(1 \mathrm{H}, \mathrm{m}), 2.05(1 \mathrm{H}, \mathrm{dd}, J=10.1,14.7), 2.15(1 \mathrm{H}, \mathrm{m}), 2.32$ $(1 \mathrm{H}, \mathrm{dd}, J=3.4,14.7), 2.32(3 \mathrm{H}, \mathrm{s}), 2.36(1 \mathrm{H}, \mathrm{t}, J=10.7), 3.06$ $(1 \mathrm{H}, \mathrm{dt}, J=3.4,11.9), 7.04-7.22(4 \mathrm{H}, \mathrm{m}) .{ }^{13} \mathrm{C} \mathrm{NMR}: 19.6\left(\mathrm{CH}_{3}\right)$, $25.9\left(\mathrm{CH}_{2}\right), 27.5\left(\mathrm{CH}_{3}\right), 28.1\left(\mathrm{CH}_{3}\right), 31.0\left(\mathrm{CH}_{2}\right), 33.7\left(\mathrm{CH}_{2}\right), 37.5$ $(\mathrm{CH}), 40.5\left(\mathrm{CH}_{2}\right), 42.1(\mathrm{CH}), 55.5(\mathrm{CH}), 79.9(\mathrm{C}), 80.2(\mathrm{C})$, $125.8(\mathrm{CH}), 125.9(\mathrm{CH}), 126.4(\mathrm{CH}), 130.0(\mathrm{CH}), 135.5(\mathrm{C})$, 142.1 (C), 171.7 (C), 173.6 (C). IR (neat): 1720. EIMS m/z: 388 $\left(\mathrm{M}^{+}\right)$, 332, 276. Anal. Calcd for $\mathrm{C}_{24} \mathrm{H}_{36} \mathrm{O}_{4}: \mathrm{C}, 74.19 ; \mathrm{H}, 9.34$. Found: C, 73.91; H, 9.47 .

4.2.5. (1R,2S,3S)-tert-Butyl 2-(tert-butoxycarbonyl)-3-(2-methylphenyl)cyclohexanacetate ( $t c-7 \mathrm{c}$ ) (Table 1, entry 2): $R_{f}=0.6$ (hexane $/ \mathrm{Et}_{2} \mathrm{O}=9 / 1$, developed twice). A colorless solid of $\mathrm{mp}$ $60-75{ }^{\circ} \mathrm{C} .42 \%$ ee (HPLC: Daicel Chiralcel OG, hexane $i$-PrOH $=500 / 1,0.5 \mathrm{~mL} / \mathrm{min}, 254 \mathrm{~nm}$; major $10.8 \mathrm{~min}$, minor $15.8 \mathrm{~min}$ ). $[\alpha]_{\mathrm{D}}{ }^{25}-8.28\left(c 0.79, \mathrm{CHCl}_{3}\right) .{ }^{1} \mathrm{H}$ NMR: $1.12(9 \mathrm{H}, \mathrm{s}), 1.19-1.27$ $(2 \mathrm{H}, \mathrm{m}), 1.46(9 \mathrm{H}, \mathrm{s}), 1.59-1.63(2 \mathrm{H}, \mathrm{m}), 1.79-1.81(2 \mathrm{H}, \mathrm{m})$, 
$2.36(3 \mathrm{H}, \mathrm{s}), 2.47(1 \mathrm{H}, \mathrm{dd}, J=4.9,15.3), 2.52(1 \mathrm{H}, \mathrm{dd}, J=3.4$ 14.7), $2.76(1 \mathrm{H}, \mathrm{m}), 2.92(1 \mathrm{H}, \mathrm{dd}, J=4.0,12.2), 3.05(1 \mathrm{H}, \mathrm{dt}, J=$ 3.7, 12.2), 7.02-7.15 (4H, m). ${ }^{13} \mathrm{C}$ NMR: $19.6\left(\mathrm{CH}_{3}\right), 20.8\left(\mathrm{CH}_{2}\right)$, $27.6\left(\mathrm{CH}_{3}\right), 28.1\left(\mathrm{CH}_{3}\right), 29.5\left(\mathrm{CH}_{2}\right), 33.1(\mathrm{CH}), 34.0\left(\mathrm{CH}_{2}\right), 34.1$ $\left(\mathrm{CH}_{2}\right), 35.3(\mathrm{CH}), 52.5(\mathrm{CH}), 80.0(\mathrm{C}), 80.2(\mathrm{C}), 124.8(\mathrm{CH})$, $125.6(\mathrm{CH}), 126.0(\mathrm{CH}), 130.1(\mathrm{CH}), 135.6(\mathrm{C}), 143.5(\mathrm{C}), 172.4$ (C), 172.9 (C). IR (Nujol): 1730, 1710. EIMS m/z: $388\left(\mathrm{M}^{+}\right), 332$, 276. Anal. Calcd for $\mathrm{C}_{24} \mathrm{H}_{36} \mathrm{O}_{4}: \mathrm{C}, 74.19 ; \mathrm{H}, 9.34$. Found: $\mathrm{C}$, 73.96; H, 9.38 .

4.2.6. (1S,2S,3S)-tert-Butyl 2-(tert-butoxycarbonyl)-3-(2-isopropylphenyl)cyclohexanacetate $(t t-7 \mathrm{~d})$ (Table 1 , entry 3): $R_{f}$ $=0.4($ hexane $/$ EtOAc $=9 / 1)$. A colorless oil. $67 \%$ ee. $[\alpha]_{\mathrm{D}}{ }^{25}$ +17.9 (c 5.11, $\left.\mathrm{CHCl}_{3}\right) .{ }^{1} \mathrm{H}$ NMR: $1.05(9 \mathrm{H}, \mathrm{s}), 1.17(3 \mathrm{H}, \mathrm{d}, J=$ 6.7), $1.25(3 \mathrm{H}, \mathrm{d}, J=6.7), 1.27-1.52(3 \mathrm{H}, \mathrm{m}), 1.46(9 \mathrm{H}, \mathrm{s}), 1.82$ $(1 \mathrm{H}, \mathrm{m}), 1.95(1 \mathrm{H}, \mathrm{m}), 2.05(1 \mathrm{H}, \mathrm{dd}, J=9.8,14.7), 2.16(1 \mathrm{H}, \mathrm{m})$, $2.31(1 \mathrm{H}, \mathrm{dd}, J=3.1,14.7), 2.41(1 \mathrm{H}, \mathrm{t}, J=11.0), 3.17(1 \mathrm{H}, \mathrm{dt}, J$ $=3.1,11.0), 3.29(1 \mathrm{H}$, septet, $J=6.7), 7.10-7.26(4 \mathrm{H}, \mathrm{m}) .{ }^{13} \mathrm{C}$ NMR: $23.2\left(\mathrm{CH}_{3}\right), 24.6\left(\mathrm{CH}_{3}\right), 26.0\left(\mathrm{CH}_{2}\right), 27.5\left(\mathrm{CH}_{3}\right), 28.12$ $(\mathrm{CH}), 28.14\left(\mathrm{CH}_{3}\right), 30.9\left(\mathrm{CH}_{2}\right), 35.0\left(\mathrm{CH}_{2}\right), 37.7(\mathrm{CH}), 40.5$ $\left(\mathrm{CH}_{2}\right), 41.3(\mathrm{CH}), 55.5(\mathrm{CH}), 79.9(\mathrm{C}), 80.2(\mathrm{C}), 124.8(\mathrm{CH})$, $125.3(\mathrm{CH}), 126.2(\mathrm{CH}), 126.9(\mathrm{CH}), 140.7(\mathrm{C}), 146.0(\mathrm{C}), 171.6$ (C), 173.7 (C). IR (neat): 1720. EIMS $m / z: 416\left(\mathrm{M}^{+}\right), 360,304$. Anal. Calcd for $\mathrm{C}_{26} \mathrm{H}_{40} \mathrm{O}_{4}: \mathrm{C}, 74.96 ; \mathrm{H}, 9.68$. Found: C, 75.17; H, 9.83. The ee was determined after reduction with $\mathrm{LiAlH}_{4}$ (see Supplementary Material).

4.2.7. (1R,2S,3S)-tert-Butyl 2-(tert-butoxycarbonyl)-3-(2-isopropylphenyl)cyclohexanacetate (tc-7d) (Table 1, entry 3): $R_{f}$ $=0.5($ hexane $/$ EtOAc $=9 / 1)$. A colorless solid of mp 79-81 ${ }^{\circ} \mathrm{C}$. $61 \%$ ee (HPLC: Daicel Chiralcel AD, hexane $/ i-\mathrm{PrOH}=100 / 1$, $0.5 \mathrm{~mL} / \mathrm{min}, 254 \mathrm{~nm}$; major $9.3 \mathrm{~min}$, minor $8.2 \mathrm{~min}) .[\alpha]_{\mathrm{D}}{ }^{25}-15.1$ (c $\left.0.56, \mathrm{CHCl}_{3}\right) .{ }^{1} \mathrm{H}$ NMR: $1.13(9 \mathrm{H}, \mathrm{s}), 1.20(3 \mathrm{H}, \mathrm{d}, J=6.7)$, $1.29(3 \mathrm{H}, \mathrm{d}, J=6.7), 1.46(9 \mathrm{H}, \mathrm{s}), 1.59-1.67(4 \mathrm{H}, \mathrm{m}), 1.79-1.82$ $(2 \mathrm{H}, \mathrm{m}), 2.52(1 \mathrm{H}, \mathrm{dd}, J=10.4,15.6), 2.57(1 \mathrm{H}, \mathrm{dd}, J=4.3$, 15.6), $2.76(1 \mathrm{H}, \mathrm{m}), 2.92(1 \mathrm{H}, \mathrm{dd}, J=4.3,11.9), 3.17(1 \mathrm{H}, \mathrm{dt}, J=$ $3.4,11.9), 3.32(1 \mathrm{H}$, septet, $J=6.7), 7.08-7.25(4 \mathrm{H}, \mathrm{m}) .{ }^{13} \mathrm{C}$ NMR: $20.7\left(\mathrm{CH}_{2}\right), 23.6\left(\mathrm{CH}_{3}\right), 24.3\left(\mathrm{CH}_{3}\right), 27.7\left(\mathrm{CH}_{3}\right), 28.0(\mathrm{CH})$, $28.1\left(\mathrm{CH}_{3}\right), 29.5\left(\mathrm{CH}_{2}\right), 33.0(\mathrm{CH}), 33.9\left(\mathrm{CH}_{2}\right), 34.5(\mathrm{CH}), 35.4$ $\left(\mathrm{CH}_{2}\right), 52.5(\mathrm{CH}), 79.8(\mathrm{C}), 80.1(\mathrm{C}), 125.0(\mathrm{CH}), 125.2(\mathrm{CH})$, $125.4(\mathrm{CH}), 126.0(\mathrm{CH}), 141.7(\mathrm{C}), 146.1(\mathrm{C}), 172.5(\mathrm{C}), 172.8$ (C). IR (Nujol): 1710. EIMS $\mathrm{m} / z$ : $416\left(\mathrm{M}^{+}\right), 360,304$. Anal. Calcd for $\mathrm{C}_{26} \mathrm{H}_{40} \mathrm{O}_{4}$ : C, 74.96; H, 9.68. Found: C, 74.69; H, 9.66.

4.2.8. $(1 S, 2 S, 3 S)$ - and $(1 R, 2 S, 3 S)$-tert-Butyl 2-(tert-butoxycarbonyl)-3-(naphthalen-1-yl)cyclohexanacetate ( $t t-7 \mathrm{e}$ and $t c-7 \mathrm{e})$ (Table 1, entry 4): $R_{f}=0.6$ (hexane/EtOAc $=9 / 1$, developed twice). A colorless oil. A 79:4 mixture of $\boldsymbol{t t}-\mathbf{7 e}$ with $81 \%$ ee and tc-7e with $39 \%$ ee. $[\alpha]_{\mathrm{D}}{ }^{25}-16.3\left(c 5.00, \mathrm{CHCl}_{3}\right) .{ }^{1} \mathrm{H}$ NMR: 0.88 $(9 \mathrm{H}, \mathrm{s}), 0.98(1.8 \mathrm{H}, \mathrm{s}), 1.21-1.50(3 \mathrm{H}, \mathrm{m}), 1.21-2.10(1.4 \mathrm{H}, \mathrm{m})$, $1.48(9 \mathrm{H}, \mathrm{s}), 1.59(1.8 \mathrm{H}, \mathrm{s}), 1.66(1 \mathrm{H}, \mathrm{m}), 1.89(1 \mathrm{H}, \mathrm{m}), 2.01(1 \mathrm{H}$, $\mathrm{m}), 2.12(1 \mathrm{H}, \mathrm{dd}, J=10.0,15.0), 2.27(1 \mathrm{H}, \mathrm{m}), 2.38(1 \mathrm{H}, \mathrm{dd}, J=$ $3.4,15.0), 2.51(0.2 \mathrm{H}, \mathrm{dd}, J=4.0,15.3), 2.55(1 \mathrm{H}, \mathrm{t}, J=11.0)$, $2.65(0.2 \mathrm{H}, \mathrm{dd}, J=11.0,15.3), 2.86(0.2 \mathrm{H}, \mathrm{m}), 3.14(0.2 \mathrm{H}, \mathrm{dd}, J$ $=4.3,11.9), 3.74(1 \mathrm{H}, \mathrm{dt}, J=3.4,11.0), 7.41-7.51(4 \mathrm{H}, \mathrm{m})$, $7.30-7.50(1 \mathrm{H}, \mathrm{m}), 7.67(0.2 \mathrm{H}, \mathrm{d}, J=7.9), 7.68(1 \mathrm{H}, \mathrm{d}, J=7.9)$, $7.81(1 \mathrm{H}, \mathrm{d}, J=8.1), 7.82(0.2 \mathrm{H}, \mathrm{d}, J=8.0), 8.16(1 \mathrm{H}, \mathrm{d}, J=8.6)$. ${ }^{13} \mathrm{C}$ NMR: $(t t-7 \mathrm{e}) 26.0\left(\mathrm{CH}_{2}\right), 27.3\left(\mathrm{CH}_{3}\right), 28.1\left(\mathrm{CH}_{3}\right), 31.2\left(\mathrm{CH}_{2}\right)$, $34.5\left(\mathrm{CH}_{2}\right), 37.9(\mathrm{CH}), 40.5\left(\mathrm{CH}_{2}\right), 40.9(\mathrm{CH}), 56.0(\mathrm{CH}), 80.0$ $(\mathrm{C}), 80.2(\mathrm{C}), 123.1(\mathrm{CH}), 123.7(\mathrm{CH}), 125.2(\mathrm{CH}), 125.3(\mathrm{CH})$, $125.7(\mathrm{CH}), 126.6(\mathrm{CH}), 128.6(\mathrm{CH}), 131.5(\mathrm{C}), 133.8(\mathrm{C}), 140.4$ (C), $171.7(\mathrm{C}), 173.1(\mathrm{C})$; $(\boldsymbol{t c}-7 \mathrm{e}) 20.9\left(\mathrm{CH}_{2}\right), 27.5\left(\mathrm{CH}_{3}\right), 29.5$ $\left(\mathrm{CH}_{2}\right), 33.4(\mathrm{CH}), 34.3\left(\mathrm{CH}_{2}\right), 34.9\left(\mathrm{CH}_{2}\right), 52.5(\mathrm{CH}), 80.1(\mathrm{C})$, $123.0(\mathrm{CH}), 125.3(\mathrm{CH}), 126.4(\mathrm{CH}), 128.8(\mathrm{CH}), 131.6(\mathrm{C})$, $133.8(\mathrm{C}), 141.8(\mathrm{C}), 172.3(\mathrm{C}), 172.8$ (C). IR (neat): 1720. EIMS $m / z: 424\left(\mathrm{M}^{+}\right), 368,312$. Anal. Calcd for $\mathrm{C}_{27} \mathrm{H}_{36} \mathrm{O}_{4}: \mathrm{C}, 76.38 ; \mathrm{H}$,
8.55. Found: C, 76.61; H, 8.64. The ee was determined after reduction with $\mathrm{LiAlH}_{4}$ (see Supplementary Material).

4.2.9. (1S,2S,3S)-tert-Butyl 2-tert-butoxycarbonyl-3-(benzo[1, 3]dioxol-5-yl)cyclohexaneacetate (tt-7f) (Table 1, entry 5): $R_{f}=$ 0.5 (hexane/ $\mathrm{Et}_{2} \mathrm{O}=4 / 1$ ). A colorless oil. $74 \%$ ee (HPLC: Daicel Chiralcel OD-H, hexane $/ i-\mathrm{PrOH}=100 / 1,1 \mathrm{~mL} / \mathrm{min}, 250 \mathrm{~nm}$; major $6.3 \mathrm{~min}$, minor $7.0 \mathrm{~min})$. $[\alpha]_{\mathrm{D}}{ }^{25}+15.2\left(c 1.24, \mathrm{CHCl}_{3}\right) .{ }^{1} \mathrm{H}$ NMR: $1.10(9 \mathrm{H}, \mathrm{s}), 1.0-1.4(3 \mathrm{H}, \mathrm{m}), 1.38(9 \mathrm{H}, \mathrm{s}), 1.70-1.78(2 \mathrm{H}$, $\mathrm{m}), 1.84(1 \mathrm{H}, \mathrm{m}), 1.95(1 \mathrm{H}, \mathrm{dd}, J=9.8,14.6), 2.03(1 \mathrm{H}, \mathrm{m}), 2.11$ $(1 \mathrm{H}, \mathrm{t}, J=11.0), 2.22(1 \mathrm{H}, \mathrm{dd}, J=2.7,14.6), 2.60(1 \mathrm{H}, \mathrm{dt}, J=$ $2.4,11.0), 5.81(2 \mathrm{H}, \mathrm{m}), 6.55(1 \mathrm{H}, \mathrm{m}), 6.60-6.63(2 \mathrm{H}, \mathrm{m}) .{ }^{13} \mathrm{C}$ NMR: $25.5\left(\mathrm{CH}_{2}\right), 27.6\left(\mathrm{CH}_{3}\right), 28.0\left(\mathrm{CH}_{3}\right), 30.7\left(\mathrm{CH}_{2}\right), 34.1$ $\left(\mathrm{CH}_{2}\right), 37.3(\mathrm{CH}), 40.3\left(\mathrm{CH}_{2}\right), 47.3(\mathrm{CH}), 56.3(\mathrm{CH}), 79.8(\mathrm{C})$, $80.0(\mathrm{C}), 100.5\left(\mathrm{CH}_{2}\right), 107.7(\mathrm{CH}), 107.9(\mathrm{CH}), 120.7(\mathrm{CH})$, 137.9 (C), 145.8 (C), 147.2 (C), 171.4 (C), 173.1 (C). IR (neat): 1728. EIMS $m / z: 418\left(\mathrm{M}^{+}\right)$. Anal. Calcd for $\mathrm{C}_{24} \mathrm{H}_{34} \mathrm{O}_{6}: \mathrm{C}, 68.87$; H, 8.19. Found: C, 69.01; H, 8.40.

4.2.10. $(1 R, 2 S, 3 S)$-tert-Butyl 2-tert-butoxycarbonyl-3-(benzo[1, 3]dioxol-5-yl)cyclohexaneacetate (tc-7f) (Table 1, entry 5): $R_{f}$ $=0.6$ (hexane $\left./ \mathrm{Et}_{2} \mathrm{O}=4 / 1\right)$. A colorless oil. $72 \%$ ee (HPLC: Daicel Chiralcel OD-H, hexane $/ i$-PrOH $=100 / 1,1 \mathrm{~mL} / \mathrm{min}, 250$ $\mathrm{nm}$; major $6.2 \mathrm{~min}$, minor $7.1 \mathrm{~min}) .[\alpha]_{\mathrm{D}}^{25}-20.1\left(\right.$ c $\left.1.78, \mathrm{CHCl}_{3}\right)$. ${ }^{1} \mathrm{H}$ NMR: $1.21(9 \mathrm{H}, \mathrm{s}), 1.2-1.4(2 \mathrm{H}, \mathrm{m}), 1.44(9 \mathrm{H}, \mathrm{s}), 1.5-1.7(2 \mathrm{H}$, $\mathrm{m}), 1.76(1 \mathrm{H}, \mathrm{m}), 1.84(1 \mathrm{H}, \mathrm{m}), 2.42(1 \mathrm{H}, \mathrm{dd}, J=9.5,15.6), 2.48$ $(1 \mathrm{H}, \mathrm{dd}, J=4.3,15.6), 2.68-2.78(3 \mathrm{H}, \mathrm{m}), 5.89(2 \mathrm{H}, \mathrm{m}), 6.64$ (1H, m), 6.69-6.71 (2H, m). ${ }^{13} \mathrm{C}$ NMR: $20.5\left(\mathrm{CH}_{2}\right), 27.8\left(\mathrm{CH}_{3}\right)$, $28.1\left(\mathrm{CH}_{3}\right), 29.5\left(\mathrm{CH}_{2}\right), 32.8(\mathrm{CH}), 34.2\left(\mathrm{CH}_{2}\right), 35.2\left(\mathrm{CH}_{2}\right), 40.5$ $(\mathrm{CH}), 52.8(\mathrm{CH}), 80.10(\mathrm{C}), 80.12(\mathrm{C}), 100.7\left(\mathrm{CH}_{2}\right), 107.8(\mathrm{CH})$, $107.9(\mathrm{CH}), 120.2(\mathrm{CH}), 139.3(\mathrm{C}), 145.6(\mathrm{C}), 147.3(\mathrm{C}), 172.4$ (C), 172.7 (C). IR (neat): 1728. EIMS m/z: $418\left(\mathrm{M}^{+}\right)$. Anal. Calcd for $\mathrm{C}_{24} \mathrm{H}_{34} \mathrm{O}_{6}: \mathrm{C}, 68.87 ; \mathrm{H}, 8.19$. Found: $\mathrm{C}, 68.66 ; \mathrm{H}, 8.22$.

\subsection{Asymmetric total synthesis of (+)- $\beta$-lycorane (Scheme 3)}

4.3.1. (1S,2S,6S)-2-(Benzo[1,3]dioxol-5-yl)-6-ethoxycarbonylmethylcyclohexanecarboxylic acid (10): To a solution of $\boldsymbol{t} t$-7a $(2.94 \mathrm{~g}, 6.0 \mathrm{mmol})$ in EtOH $(2 \mathrm{~mL})$ was added anhydrous $35 \%$ $\mathrm{HCl}$ in $\mathrm{EtOH}(20 \mathrm{~mL})$ at $\mathrm{rt}$, and the mixture was heated under reflux for $0.5 \mathrm{~h}$. Water was added at $0{ }^{\circ} \mathrm{C}$, and the whole was extracted with $\mathrm{Et}_{2} \mathrm{O}$. The organic layer was washed with brine, dried over $\mathrm{Na}_{2} \mathrm{SO}_{4}$, and concentrated. Column chromatography (hexane/EtOAc $=4 / 1)$ gave the title compound $(2.03 \mathrm{~g}, 98 \%)$ as colorless needles of mp 90-91 ${ }^{\circ} \mathrm{C}: R_{f}=0.4$ (hexane/EtOAc $=4 / 1$ ). $[\alpha]_{\mathrm{D}}{ }^{25}+39.6\left(c 1.00, \mathrm{CHCl}_{3}\right) .{ }^{1} \mathrm{H}$ NMR: $1.11(1 \mathrm{H}, \mathrm{m}), 1.23(3 \mathrm{H}, \mathrm{t}$, $J=7.0), 1.36-1.51(2 \mathrm{H}, \mathrm{m}), 1.82-1.90(3 \mathrm{H}, \mathrm{m}), 2.11-2.19(2 \mathrm{H}$, $\mathrm{m}), 2.26(1 \mathrm{H}, \mathrm{t}, J=11.0), 2.36(1 \mathrm{H}, \mathrm{m}), 2.71(1 \mathrm{H}, \mathrm{dt}, J=2.8$, 11.0), $4.09(2 \mathrm{H}, \mathrm{q}, J=7.0), 5.89(2 \mathrm{H}, \mathrm{s}), 6.59(1 \mathrm{H}, \mathrm{dd}, J=1.5$, 7.9), 6.64-6.68 (2H, m). ${ }^{13} \mathrm{C}$ NMR: $14.1\left(\mathrm{CH}_{3}\right), 25.4\left(\mathrm{CH}_{2}\right), 30.9$ $\left(\mathrm{CH}_{2}\right), 33.7\left(\mathrm{CH}_{2}\right), 37.0(\mathrm{CH}), 39.4\left(\mathrm{CH}_{2}\right), 46.6(\mathrm{CH}), 55.7(\mathrm{CH})$, $60.4\left(\mathrm{CH}_{2}\right), 100.7\left(\mathrm{CH}_{2}\right), 107.4(\mathrm{CH}), 108.0(\mathrm{CH}), 120.4(\mathrm{CH})$, 137.4 (C), 146.1 (C), 147.5 (C), 172.1 (C), 179.6 (C). IR (Nujol): 2800-3300, 1720, 1685. EIMS m/z: $334\left(\mathrm{M}^{+}\right), 288,201,135$. Anal. Calcd for $\mathrm{C}_{18} \mathrm{H}_{22} \mathrm{O}_{6}: \mathrm{C}, 64.66 ; \mathrm{H}, 6.63$. Found: C, 64.61; $\mathrm{H}$, 6.55 .

4.3.2. (4S,4aR,11bR)-Ethyl (6-0xo-1,2,3,4,4a,5,6,11b-octahydro[1,3]dioxolo[4,5-j]phenanthridin-4-yl)acetate (11): To a solution of $10(1.84 \mathrm{~g}, 5.5 \mathrm{mmol})$ in toluene $(16 \mathrm{~mL})$ were added diphenylphosphoryl azide $(1.3 \mathrm{~mL}, 6 \mathrm{mmol})$ and $\mathrm{Et}_{3} \mathrm{~N}(0.92 \mathrm{~mL}$, $6.6 \mathrm{mmol}$ ) at $0{ }^{\circ} \mathrm{C}$. The whole was heated under reflux for $0.5 \mathrm{~h}$. After cooled to rt, the mixture was concentrated in vacuo to give colorless oil, which indicated the absorbance at $2250 \mathrm{~cm}^{-1}$ in the IR spectrum. To the above oil was added PPA $(10 \mathrm{~mL})$ at $0{ }^{\circ} \mathrm{C}$, and the whole was stirred for $0.5 \mathrm{~h}$ at $\mathrm{rt}$. Water was added, and the mixture was extracted with $\mathrm{CHCl}_{3}$. The organic layer was successively washed with water, saturated $\mathrm{NaHCO}_{3}$, and brine, 
dried over $\mathrm{Na}_{2} \mathrm{SO}_{4}$, and concentrated. Column chromatography $\left(\mathrm{CHCl}_{3} / \mathrm{EtOAc}=4 / 1\right)$, followed by recrystallization from EtOH gave the title compound $(1.28 \mathrm{~g}, 70 \%)$ as colorless needles of $\mathrm{mp}$ $205-206{ }^{\circ} \mathrm{C}: R_{f}=0.3\left(\mathrm{CHCl}_{3} / \mathrm{EtOAc}=4 / 1\right) .[\alpha]_{\mathrm{D}}{ }^{25}+72.7(c 1.06$, $\left.\mathrm{CHCl}_{3}\right) .{ }^{1} \mathrm{H}$ NMR: $1.17-1.36(2 \mathrm{H}, \mathrm{m}), 1.29(3 \mathrm{H}, \mathrm{t}, J=7.0), 1.52$ $(1 \mathrm{H}, \mathrm{m}), 1.90-1.94(2 \mathrm{H}, \mathrm{m}), 2.05(1 \mathrm{H}, \mathrm{m}), 2.27(1 \mathrm{H}, \mathrm{dd}, J=7.0$, 15.9), $2.34(1 \mathrm{H}, \mathrm{m}), 2.60(1 \mathrm{H}, \mathrm{dd}, J=5.0,15.9), 2.71(1 \mathrm{H}, \mathrm{dt}, J=$ $3.7,11.6), 3.03(1 \mathrm{H}, \mathrm{t}, J=11.6), 4.19(2 \mathrm{H}, \mathrm{q}, J=7.0), 6.00(2 \mathrm{H}$, $\mathrm{m}), 6.73(1 \mathrm{H}$, brs $), 6.76(1 \mathrm{H}, \mathrm{s}), 7.51(1 \mathrm{H}, \mathrm{s}) \cdot{ }^{13} \mathrm{C} \mathrm{NMR}: 14.1$ $\left(\mathrm{CH}_{3}\right), 24.5\left(\mathrm{CH}_{2}\right), 26.9\left(\mathrm{CH}_{2}\right), 31.3\left(\mathrm{CH}_{2}\right), 37.7(\mathrm{CH}), 37.8\left(\mathrm{CH}_{2}\right)$, $41.6(\mathrm{CH}), 59.6(\mathrm{CH}), 60.7\left(\mathrm{CH}_{2}\right), 101.4\left(\mathrm{CH}_{2}\right), 103.9(\mathrm{CH})$, $107.9(\mathrm{CH}), 123.2(\mathrm{C}), 138.0(\mathrm{C}), 146.4(\mathrm{C}), 151.0(\mathrm{C}), 165.8(\mathrm{C})$ 172.6 (C). IR (Nujol): 3200, 1735, 1660. EIMS m/z: $331\left(\mathrm{M}^{+}\right)$, 243, 216, 202. Anal. Calcd for $\mathrm{C}_{18} \mathrm{H}_{21} \mathrm{NO}_{5}: \mathrm{C}, 65.24 ; \mathrm{H}, 6.39 ; \mathrm{N}$, 4.23. Found: C, 65.02; H, 6.34; N, 4.25.

4.3.3. (+)- $\boldsymbol{\beta}$-Lycorane (2b): To a solution of $11(662 \mathrm{mg}, 2.0$ $\mathrm{mmol})$ in THF $(100 \mathrm{~mL})$ was added $\mathrm{BH}_{3} \cdot \mathrm{SMe}_{2}(10 \mathrm{M} ; 1.0 \mathrm{~mL}, 10$ mmol) at $0{ }^{\circ} \mathrm{C}$, and the mixture was heated under reflux for $3 \mathrm{~d}$. To the mixture was added $10 \% \mathrm{NaOH}$ at $0{ }^{\circ} \mathrm{C}$, and the whole was extracted with toluene. The organic layer was washed with brine, dried over $\mathrm{Na}_{2} \mathrm{SO}_{4}$, and concentrated. Column chromatography $(\mathrm{EtOAc} / \mathrm{EtOH}=4 / 1)$ gave the title compound $(442 \mathrm{mg}, 70 \%)$ as colorless plates of mp $72-73{ }^{\circ} \mathrm{C}$ (lit. ${ }^{3,22} \mathrm{mp} 72-73{ }^{\circ} \mathrm{C}$ ): $R_{f}=0.4$ $(\mathrm{EtOAc} / \mathrm{EtOH}=4 / 1) .[\alpha]_{\mathrm{D}}{ }^{20}+153(c 1.02, \mathrm{EtOH}) ;$ lit. $[\alpha]_{\mathrm{D}}{ }^{20}-$ $143.3(c 1.04, \mathrm{EtOH})$ for the enantiomer, ${ }^{3}[\alpha]_{\mathrm{D}}{ }^{20}+142.5(c 0.17$, EtOH $).{ }^{22} \mathrm{H}_{\mathrm{NMR}}$ : 1.1-1.2 (2H, m) $1.35-1.55(2 \mathrm{H}, \mathrm{m}), 1.51(1 \mathrm{H}$ $\mathrm{t}, J=10.5), 1.65(1 \mathrm{H}, \mathrm{m}), 1.9-2.05(3 \mathrm{H}, \mathrm{m}), 2.26-2.35(2 \mathrm{H}, \mathrm{m})$, $2.50(1 \mathrm{H}, \mathrm{t}, J=10.5), 3.33(1 \mathrm{H}, \mathrm{d}, J=14.3), 3.38(1 \mathrm{H}, \mathrm{dt}, J=5.5$ 9.0), $4.06(1 \mathrm{H}, \mathrm{d}, J=14.3), 5.87(1 \mathrm{H}, \mathrm{brs}), 5.88$ (1H, brs), 6.50 $(1 \mathrm{H}, \mathrm{s}), 6.71(1 \mathrm{H}, \mathrm{s}) .{ }^{13} \mathrm{C}$ NMR: $26.4\left(\mathrm{CH}_{2}\right), 28.2\left(\mathrm{CH}_{2}\right), 28.8$ $\left(\mathrm{CH}_{2}\right), 30.0\left(\mathrm{CH}_{2}\right), 41.7(\mathrm{CH}), 42.9(\mathrm{CH}), 53.8\left(\mathrm{CH}_{2}\right), 57.2\left(\mathrm{CH}_{2}\right)$, $71.8(\mathrm{CH}), 100.6\left(\mathrm{CH}_{2}\right), 105.3(\mathrm{CH}), 106.8(\mathrm{CH}), 128.4(\mathrm{C})$, 131.2 (C), 145.6 (C), 146.1 (C). IR $\left(\mathrm{CHCl}_{3}\right): 2928,1593,1485$. EIMS $m / z: 257\left(\mathrm{M}^{+}\right) .{ }^{1} \mathrm{H}$ NMR and ${ }^{13} \mathrm{C}$ NMR are in good agreement with those reported in ref $6 c$ and $6 b$, respectively, while there are slight differences in ${ }^{1} \mathrm{H}$ NMR and IR from those reported in ref $6 \mathrm{~d}$, probably due to a misprint; ours $1.9-2.05(3 \mathrm{H}$, $\mathrm{m}$ ) instead of reported $1.93-1.98(2 \mathrm{H}, \mathrm{m})$ and $3.23(1 \mathrm{H}, \mathrm{m})$, and ours $1593 \mathrm{~cm}^{-1}$ instead of reported $1724 \mathrm{~cm}^{-1}$, respectively.

\section{Acknowledgment}

We thank JSPS and MEXT for financial support.

\section{Supplementary Material}

Determination of enantiomeric excess, and relative and absolute configuration, and characterization data of the products (PDF).

\section{References and notes}

1 (a) Jin, Z. Nat. Prod. Rep. 2011, 28, 1126-1142. (b) Jin, Z. Nat. Prod. Rep. 2009, 26, 363-381. (c) Zarotsky, V.; Sramek, J. J.; Cutler, N. R. Am. J. Health-Syst. Pharm. 2003, 60, 446-452. (d) WHO Drug Info. 1998, 12, 205.

2 (a) Cook, J. W.; Loudon, J. D. In The Alkaloids; Manske, R. H. F., Holmes, H. L., Eds; Academic Press: New York, 1952; Vol. 2, pp $331-$ 352 (b) Kondo, $\mathrm{H}$; Uyeo, S Chem. Ber 1935, 68, 1756-1760.

Kotera, K. Tetrahedron 1961, 12, 240-247.

4 Yamada, K.; Yamashita, M.; Sumiyoshi, T.; Nishimura, K.; Tomioka, K. Org. Lett. 2009, 11, 1631-1633.

5 Recent synthetic studies of lycorine: Sun, Z.; Zhou, M.; Li, X.; Meng, X.; Peng, F.; Zhang, H; Shao, Z. Chem. Eur. J. 2014, 20, 6112-6119. Other approaches are cited therein.

6 Racemic syntheses: (a) Hill, R. K.; Joule, J. A.; Loeffler, L. J. J. Am. Chem. Soc. 1962, 84, 4951-4956. (b) Martin, S. F.; Tu, C.-y. J. Org Chem 1981, 46, 3764-3767. (c) Martin, S. F.; Tu, C.-y.; Kimura, M. Simonsen, S. H. J. Org. Chem. 1982, 47, 3634-3643. Asymmetric syntheses: (d) Dong, L.; Xu, Y.-J.; Yuan, W.-C.; Cui, X.; Cun, L.-F.; Gong,
L.-Z. Eur. J. Org. Chem. 2006, 4093-4105. (e) Hong, B.-C.; Nimje, R. Y.; Wu, M.-F.; Sadani, A. A. Eur. J. Org. Chem. 2008, 1449-1457.

7 Our other approaches for lycoranes: (a) Yasuhara, T.; Nishimura, K.; Yamashita, M.; Fukuyama, N.; Yamada, K.; Muraoka, O.; Tomioka, K. Org. Lett. 2003, 5, 1123-1126. (b) Yasuhara, T.; Osafune, E.; Nishimura, K.; Yamashita, M.; Yamada, K.; Muraoka, O.; Tomioka, K. Tetrahedron Lett. 2004, 45, 3043-3045.

8 Our total syntheses of other Amaryllidaceae alkaloids: (a) Tomioka, K.; Koga, K.; Yamada, S. Chem. Pharm. Bull. 1977, 25, 2681-2688. (b) Shimizu, K.; Tomioka, K.; Yamada, S. Koga, K. Chem. Pharm. Bull. 1978, 26, 3765-3771. (c) Yamada, K.; Mogi, Y.; Mohamed, M. A.; Takasu, K.: Tomioka, K. Org. Lett. 2012, 14, 5868-5871.

9 (a) Tomioka, K. Synthesis 1990, 541-549. (b) Kanai, M.; Shibasaki, M. In Catalytic Asymmetric Synthesis; Ojima, I., ed: VCH: Weinheim, 2000, p. 569-592. (c) Sibi, M. P.; Manyem, S. Tetrahedron 2000, 56 , 8033-8061. (d) Krause, N.; Hoffmann-Röder, A. Synthesis 2001, 171196. (e) Feringa, B. L.; Naasz, R.; Imbos, R.; Arnold, L. A. In Modern Organocopper Chemistry; Krause, N., ed.; Wiley-VCH: Weinheim, 2002, chapter 7. (f) Hayashi, T.; Yamasaki, K. Chem. Rev. 2003, 103, 2829-2844. (g) Tomioka, K. In Comprehensive Asymmetric Catalysis, Supplement; Jacobsen, E. N., Pfaltz, A., Yamamoto, H., Eds.; Springer, 2004; Chapter 31.1, pp 109-124. (h) Yamataka, H.; Yamada, K.; Tomioka, K. In The Chemistry of Organolithium Compounds; Rappoport, Z., Marek, I., Eds; Wiley, 2004; Part 1, Chapter 14. (i) Christoffers, J.; Koripelly, G.; Rosiak, A.; Rössle, M. Synthesis 2007, 1279-1300. (j) Tomioka, K.; Yamamoto, Y.; Yamada, K. In Comprehensive Chirality; Carreira, E. M., Yamamoto, H., Eds.; Elsevier, 2012; Vol. 4, pp 122-131.

10 (a) Asano, Y.; Iida, A.; Tomioka, K. Tetrahedron Lett. 1997, 38, 89738976. (b) Asano, Y.; Iida, A.; Tomioka, K. Chem. Pharm. Bull. 1998 46, 184-186. (c) Asano, Y: Yamashita, M.; Nagai, K.; Kuriyama, M.; Yamada, K.; Tomioka, K. Tetrahedron Lett. 2001, 42, 8493-8495.

11 Diastereoselective conjugate addition cascades of dienedioates: (a) Saito, S.; Hirohara, Y.; Narahara, O.; Moriwake, T. J. Am. Chem. Soc. 1989, 111, 4533-4535. (b) Saito, S.; Hara, T.; Naka, K.; Hayashi, T.; Moriwake, T. Synlett 1992, 241-243. (c) Klimko, P. G.; Singleton, D. A. J. Org. Chem. 1992, 57, 1733-1740. (d) Uyehara, T.; Shida, N.; Yamamoto, Y. J. Org. Chem. 1992, 57, 3139-3145. (e) Shida, N.; Uyehara, T.; Yamamoto, Y. J. Org. Chem. 1992, 57, 5049-5051. (f) Yoshii, E.; Hori, K.; Nomura, K.; Yamaguchi, K. Synlett 1995, 568-571. (g) Urones, J. G.; Garrido, N. M.; Díez, D.; Dominguez, S. H.; Davies, S. G. Tetrahedron: Asymmetry 1997, 8, 2683-2685. (h) Davies, S. G.; Díez, D.; Dominguez, S. H.; Garrido, N. M.; Kruchinin, D.; Price, P. D.; Smith, A D. Org. Biomol. Chem. 2005, 3, 1284-1301.

12 Our other approaches of conjugate addition-triggered cascade reactions: (a) Nagaoka, Y.; Tomioka, K. Org. Lett. 1999, 1, 1467-1469. (b) Ono, M.; Nishimura, K.; Nagaoka, Y.; Tomioka, K. Tetrahedron Lett. 1999, 40, 6979-6982. (c) Ono, M.; Nishimura, K.; Tsubouchi, H.; Nagaoka, Y.; Tomioka, K. J. Org. Chem. 2001, 66, 8199-8203. (d) Nagaoka, Y.; El-Koussi N.; Uesato S.; Tomioka, K. Tetrahedron Lett. 2002, 43 , 4355-4359. (e) Inoue, H.; Nagaoka, Y.; Tomioka, K. J. Org. Chem. 2002, 67, 5864-5867. (f) Nishimura, K.; Tsubouchi, H.; Ono, M.; Hayama, T.; Nagaoka Y.; Tomioka, K. Tetrahedron Lett. 2003, 44 , 2323-2326. (g) Sakai, T.; Kawamoto, Y.; Tomioka, K. J. Org. Chem. 2006, 71, 4706-4709. (h) Suzuki, M.; Kawamoto, Y.; Sakai, T.; Yamamoto, Y.; Tomioka, K. Org. Lett. 2009, 11, 653-655. (i) Yamada, K.; Maekawa, M.; Akindele, T.; Yamamoto, Y.; Nakano, M.; Tomioka, K. Tetrahedron 2009, 65, 903-908. (j) Yamamoto, Y.; Yasuda, Y.; Nasu, H. Tomioka, K. Org. Lett. 2009, 11, 2007-2009. (k) Yamada, K.; Konishi, T.; Nakano, M.; Fujii, S.; Cadou, R.; Yamamoto, Y.; Tomioka, K. J. Org. Chem. 2012, 77, 5775-5780. (1) Harada, S.; Sakai, T.; Takasu, K.; Yamada, K.; Yamamoto, Y.; Tomioka, K. Chem. Asian J. 2012, 7, 2196-2198. (m) Harada, S.; Sakai, T.; Takasu, K.; Yamada, K.; Yamamoto, Y : Tomioka, K. J. Org. Chem 2012, 77, 7212-7222. (n) Harada, S.; Sakai, T.; Takasu, K.; Yamada, K.; Yamamoto, Y.; Tomioka, K. Tetrahedron 2013, 69, 3264-3273.

13 NMR and X-ray studies on substituted phenyllithiums: (a) Reich, H. J.; Goldenberg, W. S.; Sanders, A. W.; Jantzi, K. L.; Tzschucke, C. C. J. Am. Chem. Soc. 2003, 125, 3509-3521. (b) Reich, H. J.; Goldenberg, W. S.; Gudmundsson, B. Ö.; Sanders, A. W.; Kulicke, K. J.; Simon, K.; Guzei, I. A. J. Am. Chem. Soc. 2001, 123, 8067-8079. (c) Boman, A.; Johnels, D. Magn. Reson. Chem. 2000, 38, 853-860.

14 Doi, H.; Sakai, T.; Iguchi, M.; Yamada, k.; Tomioka, K. J. Am. Chem. Soc., 2003, 125, 2886-2887.

15 Also in the $\mathrm{LiAlH}_{4}$ reduction of $\boldsymbol{t t}-\mathbf{7 b}$, the ester group at the C-2 remained unchanged (see Supplementary Material).

16 Ninomiya, K.; Shioiri, T.; Yamada, S. Tetrahedron 1974, 30, 21512157.

17 (a) Sánchez I. H.; Mendoza M. T. Tetrahedron Lett. 1980, 21, 36513654. (b) Taylor, E. C., Jr.; Kalenda, N. W. J. Am. Chem. Soc. 1954, 76, 1699-1700.

18 Venuti, M. C.; Ort, O. Synthesis 1988, 985-988.

19 Shindo, M.; Koga, K.; Tomioka, K. J. Org. Chem. 1998, 63, 9351-9357.

20 Davies, G.; Díez, D.; Dominguez, S. H.; Garrido, N. M.; Kruchinin, D.; Price, P. D.; Smith, A. D. Org. Biomol. Chem. 2005, 3, 1284-1301.

21 Mattson, R. J.; Sloan, C. P.; Lockhart, C. C.; Catt, J. D.; Gao, Q.; Huang, S. J. Org. Chem. 1999, 64, 8004-8007.

22 Dong, L.; Xu, Y.-J.; Yuan, W.-C.; Cui, X.; Cun, L.-F.; Gong, L.-Z. Eur. J. Org. Chem. 2006, 4093-4105. 